\title{
Reuna
}

\section{COOPERAÇÃO E APRENDIZADO INTERORGANIZACIONAL PELO USO DE REDES SOCIAIS DIGITAIS: UMA ANÁLISE NO ARRANJO PRODUTIVO LOCAL (APL) DE TECNOLOGIA DA INFORMAÇÃO EM ARACAJU/SE}

\author{
COOPERATION AND INTERORGANIZATIONAL LEARNING FOR THE USE OF \\ DIGITAL SOCIAL NETWORKS: AN ANALYSIS OF LOCAL PRODUCTIVE \\ ARRANGEMENT (APL) OF INFORMATION TECHNOLOGY IN ARACAJU/SE
}

http://dx.doi.org/10.21714/2179-8834/2019v24n3p20-40

Ronalty Oliveira Rocha

Universidade Federal de Sergipe (UFSE), Brasil.

E-mail: ronaltyrocha@gmail.com

Maria Elena Leon Olave
Universidade Federal de Sergipe (UFSE), Brasil.
E-mail: mleonolave@gmail.com

Submissão: 24 Nov. 2018 Publicação: 02 Nov. 2019. Sistema de avaliação: Double blind review. Centro Universitário UNA, Belo Horizonte - MG, Brasil. Editor geral: Prof. Dr. Gustavo Quiroga Souki

Este artigo encontra-se disponível nos seguintes endereços eletrônicos:

http://revistas.una.br/index.php/reuna/article/view/1055

http://dx.doi.org/10.21714/2179-8834/2019v24n3p20-40

\section{Resumo}

$\mathrm{O}$ presente estudo analisa como as redes sociais digitais (RSD) promovem a cooperação e aprendizado no arranjo produtivo local (APL) de tecnologia da informação (TI) de Aracaju/SE. A pesquisa consiste em um estudo qualitativo descritivo com uso de estudo de casos múltiplos no APL de TI de Aracaju/SE. Para coleta dos dados foi aplicado um roteiro de entrevista semiestruturada a três diferentes gestores (sócios) de empresas participantes do APL. Os resultados indicam que as empresas utilizam redes sociais digitais como o Facebook, WhatsApp, Telegram e Google Group para troca de informações técnicas em uma cooperação incipiente, com baixo aprendizado interorganizacional e inexpressivos ganhos competitivos. Em função dos resultados apresentados, e em consideração ao potencial colaborativo das empresas pesquisadas, esse estudo propôs um modelo conceitual para cooperação e aprendizado pelo uso de redes sociais digitais.

Palavras-chave: Arranjos produtivos locais. Cooperação. Aprendizado interorganizacional. Redes sociais digitais. 


\section{Abstract}

The present study analyzes how digital social networks promote cooperation and learning in the Aracaju / SE local information technology (APL) productive arrangement. The research consists of a qualitative descriptive study using multiple case study in Aracaju / SE IT APL. To collect the data, a script of a semi-structured interview was applied to three different managers of companies participating in the APL. The results indicate that companies use digital social networks like Facebook, WhatsApp, Telegram and Google Group to exchange technical information in an incipient cooperation, with low interorganizational learning and inexpressive competitive gains. Based on the results presented, and considering the collaborative potential of the companies surveyed, this study proposed a conceptual model for cooperation and learning through the use of digital social networks.

Keywords: Local productive arrangements. Cooperation. Interorganizational learning. Digital social networks.

\section{Introdução}

Os arranjos produtivos locais (APLs) têm cada vez mais ganhado relevância e importância de políticas públicas e ação empreendedores em razão dos diferentes benefícios proporcionados às empresas participantes. Genericamente, os arranjos produtivos locais são aglomerações geográficas entre empresas e atores sociais, políticos e econômicos que se aproveitam da proximidade geográfica para alcançar benefícios resultantes do trabalho colaborativo (CARRIEL, 2013).

Aliás, um APL organizado e bem estruturado, com apropriada cooperação do empresariado da localidade, promove progresso vultoso para a região em que está incluído, e consequentemente atribui maior resistência e competitividade às empresas participantes (BALESTRIN; VERSCHOORE, 2008).

É de ressaltar que o progresso resultante da cooperação empresarial em APLs é diretamente influenciado pelo sucesso dos processos de aprendizagem interorganizacional nesses arranjos. Colet (2016) explica que o aprendizado interorganizacional consiste em destacada estratégia que agrega vantagem competitiva para as organizações, em especial para as empresas de pequeno porte, fomentando a criação de novos conhecimentos, soluções e inovações.

Destaca-se que o aprendizado interorganizacional está fundamentado, principalmente, na comunicação empresarial, e os mecanismos para essa comunicação, com o passar dos anos, foram aperfeiçoados pela inserção de ferramentas da tecnologia da informação, tais como as redes sociais digitais, que outorgaram maior agilidade, flexibilidade e velocidade na comunicação (SÁNCHEZ; MUIÑA, 2011), permitindo as organizações coletar e compartilhar grandes quantidades de dados com os seus parceiros, promovendo, assim, práticas de cooperação e aprendizado interorganizacional, simultaneamente (PARK; STYLIANOU; SUBRAMANIAM, 2015).

Inclusive, segundo a pesquisa Digital in 2016, da We Are Social, realizada ao longo do último trimestre de 2015 , no Brasil, em média $45 \%$ da população está ativa em redes sociais de todos os tipos. O Facebook, por exemplo, que foi criado em 2004 
para ser uma rede de contatos entre universitários, tornou-se rapidamente em um dos sites de relacionamento mais destacados, sendo inclusive a rede social mais acessada no Brasil em 2015 (DIGITAL IN 2016). Já o WhatsApp, segunda colocada, é utilizada por $76 \%$ dos assinantes móveis no Brasil, segundo dados da Mobile Ecosystem Forum (2016).

A expressividade dos dados sobre o uso de redes sociais digitais no Brasil instiga a investigação acerca da utilização dessas ferramentas para cooperação e aprendizado entre empresas, especialmente entre as empresas do setor de TI que movimentaram $R \$ 59,9$ bilhões de reais e geraram 120.000 empregos diretos em 2015 (ABES, 2016). Em Aracaju/SE, os últimos dados revelados indicam que as empresas de TI faturavam $R \$ 60$ milhões de reais em 2010 (SEDETEC, 2011) e foram responsáveis pela ocupação de 929 postos de trabalho em 2015 (MTE/RAIS, 2016).

Apesar da relevância e potencialidade das redes sociais digitais para promoção da cooperação e aprendizado em APLs, não foram identificados na literatura nacional estudos que contemplem a utilização explicita dessas ferramentas para cooperação. Em geral, a maioria dos trabalhos buscar demonstrar as vantagens associadas à cooperação e os benefícios advindos do aprendizado interfirmas. Nesse sentido, parece haver uma lacuna no que se refere ao uso de redes sociais digitais como facilitadoras da cooperação e aprendizado, especialmente entre empresas de TI.

A fim de compreender a conexão entre cooperação, aprendizado e redes sociais digitais esse estudo teve como objetivo analisar como o uso de redes sociais digitais (RSD) tem colaborado para praticas cooperativas e aprendizado no arranjo produtivo local de Tecnologia da informação (APL) de Aracaju/SE. Para tal intento, fez-se a escolha pelo método de estudo de casos múltiplos e na fase de coleta dos dados foi utilizado um roteiro de entrevistas semiestruturado em três empresas de TI participantes do APL de TI da grande Aracaju. Os dados foram examinados por meio da análise descritiva das evidências identificadas.

Do ponto de vista teórico essa pesquisa reforça os estudos sobre a importância da cooperação e aprendizado em APLs, assim como identifica as vantagens e possibilidades associadas ao uso de redes sociais digitais em arranjos produtivos locais. Além disso, este estudo propõe um modelo conceitual de cooperação e aprendizado pelo uso de redes sociais digitais. Do ponto de vista prático essa pesquisa tende a demonstrar aos atores de arranjos produtivos locais a relevância da comunicação e geração de conhecimentos por redes sociais digitais, assim como alertar aos mesmos da subutilização das potencialidades dessas ferramentas.

\section{Cooperação em arranjos produtivos locais (APL)}

Cassiolato, Lastres e Maciel (2003) apresentam que APLs são aglomerações territoriais de agentes econômicos, políticos e sociais, focados em um determinado segmento de atividades econômicas, nas quais podem surgir, ainda que incipientes, vínculos de interpendência para produção, interação, cooperação e aprendizagem, assim como para favorecer a inserção de inovação em produtos, processos e formas de organizações (TISSOT et al., 2016). 
Monteiro, Noronha e Paulo Neto (2011) defendem que algumas características são fundamentais para compreensão dos APLs, são elas: concentração geográfica; especialização produtiva; multiplicidade de atores como empresas, universidades, autoridades públicas e outras instituições relacionadas; competição e cooperação (características da relação entre os diferentes atores do APL); massa crítica (para gerar dinâmica interna) e inovação (as organizações dentro de um APL devem estar envolvidas em processos de mudança tecnológica, comercial e organizacional).

Tisott et al., (2016) acrescentam que a participação em arranjos produtivos locais proporciona as empresas, especialmente as de pequeno e médio porte, transposição às barreiras de crescimento, por proporcionar maior eficiência produtiva e aumentar a capacidade de comercialização de produtos no mercado nacional e/ou internacional.

Ainda sobre aglomeração de empresas, Silva e Muylder (2015) evidenciam que um dos principais papeis dos APLs é alavancar a cooperação e interação entre empresas que atuam em um mesmo segmento econômico e que estão estabelecidas em uma determinada região.

Por falar em cooperação, especialmente em APls, essa pode ser entendida como o trabalho conjunto em atividades produtivas e/ou de gestão entre parceiros comerciais / institucionais, e entre pessoas e organizações que se associam, se complementam e se desenvolvem para alcançar benefícios mútuos e objetivos reciprocamente compatíveis que seriam onerosos e inexecutáveis individualmente (BRITO; BRITO; HASHIBA, 2014; PINTO; CRUZ; COBE, 2015).

É importante destacar que para cooperar e efetivamente combinar seus recursos, as empresas parceiras, em APLs ou não, precisam se alinhar, porque geralmente essas empresas têm diferentes características, metas, valores, culturas, estratégias, estilos de gestão e abordagens operacionais (AUSTIN; SEITANIDI, 2012). Além disso, é necessário que haja confiança interorganizacional para reforçar a cooperação de parceiros, dissolver conflitos potenciais, promover a partilha de informações e reforçar orientações para parcerias de longo prazo (CHAN; CHONG; ZHOU, 2012).

Silveira e Farina (2012) completam destacando que o sucesso da cooperação entre empresas em APLs deve abarcar itens essenciais, tais quais: aprendizado e inovação; governança; alianças estratégicas; integração de competências; construção social com redes de relacionamentos pessoais; capacidade de comunicação; e interesses compartilhados.

Assim, Esteves (2012) sintetiza que a cooperação, em termos de gênese conceitual, é construída, fundamentalmente, sob três aspectos: (1) Estratégico - as empresas que cooperam devem ter objetivos estratégicos comuns para obtenção de vantagens competitivas; (2) Econômicos - intenção por partilhar recursos e reduzir riscos financeiros; e (3) Humanos sociais - necessidade de confiança mútua entre os participantes para que a cooperação se operacionalize, de fato.

O Quadro 1 resume os principais benefícios resultantes da cooperação em APLs. 
Quadro 1 - Benefícios da cooperação em APLs

\begin{tabular}{|l|l|}
\hline \multicolumn{1}{|c|}{ Autor } & \multicolumn{1}{c|}{ Benefícios } \\
\hline \multirow{4}{*}{ Vershoore e Balestrin (2008) } & Ganhos de escala e poder de mercado \\
\cline { 2 - 2 } & Acesso a soluções técnico/financeiras \\
\cline { 2 - 2 } & Aprendizagem \\
\cline { 2 - 2 } & Inovação \\
\cline { 2 - 2 } Balestrin, Verschoore e Reyes JR. & Redução de custos e riscos \\
\cline { 2 - 2 }$(\mathbf{2 0 1 0 )}$ & Robustez de relações sociais \\
\hline \multirow{4}{*}{ Martins, Farias e Farina (2016) } & Intercâmbio deliberado de conhecimentos \\
\cline { 2 - 2 } & Compartilhamento e codesenvolvimento de produtos \\
\cline { 2 - 2 } & Criação e identificação de novos mercados \\
\hline & Maior flexibilidade. \\
\cline { 2 - 2 } & Acesso e compartilhamento de informações. \\
\cline { 2 - 2 } & $\begin{array}{l}\text { Compartilhamento de recursos financeiros, } \\
\text { mercadológicos e tecnológicos. }\end{array}$ \\
\cline { 2 - 2 } & Equilíbrio mercadológico. \\
\cline { 2 - 2 } & Sobrevivência empresarial. \\
\hline
\end{tabular}

Fonte: Elaborado pelos autores.

Enfim, nas relações de cooperação, cada empresa se concentra em suas próprias competências essenciais, e as várias empresas podem complementar as competências essenciais umas das outras, aprendendo entre si (PARK; SUBRAMANIAM; STYLIANOU, 2015). Nessa perspectiva, ressalta-se que o sucesso das redes de cooperação está fundamentado nos laços sociais que se estabelecem entre os diferentes agentes envolvidos, assim como no fluxo de informações, conhecimentos e aprendizado entre empresas parceiras (JHON et al., 2016). Dada a relevância da transferência de conhecimentos e aprendizado a partir de atividades cooperadas, esse tema será explorado no tópico a seguir.

\section{Redes sociais e aprendizagem interorganizacional}

A aprendizagem interorganizacional refere-se ao processo dinâmico de geração, acumulação, distribuição e uso do conhecimento entre organizações que estão proativamente cooperantes entre si (MOZZATO; BITTENCOURT, 2014).

Coghlan e Coughlan (2015) defendem que um dos interesses iniciais de empresas que adotam práticas de cooperação interorganizacional é explorar fontes externas de conhecimento especializado. Assim, através do trabalho conjunto, estas firmas se tornam capazes de criar novos conhecimentos, melhorar suas práticas de trabalho, e ampliar seu leque de habilidades e conhecimentos técnicos (PANJAITAN; NOORDERHAVEN, 2008; GIBB; SUNE; ALBERS, 2016).

Nesse contexto, Silva e Muylder (2015) complementam que a interação entre os atores de um APL sucinta um ambiente propício ao compartilhamento de informações, conhecimentos e habilidades que alavancam os processos de inovação, competitividade, eficiência coletiva, e aprendizado.

Para Castro (2009) o aprendizado interorganizacional em APLs é promovido pela troca de informações produtivas, tecnológicas e mercadológicas, assim como 
pela realização de ações reciprocas entre empresas e outros atores participantes do APL (universidades, instituições financeiras, órgãos governamentais, dentre outros). Os autores ainda apontam que a associação e interação de diversas competências organizacionais e individuais fomenta o compartilhamento de conhecimentos. Entretanto, para que haja interação, aprendizado e inovação, é preciso que haja cooperação (OURO FILHO; OLAVE; BARRETO, 2015) para que as empresas participantes concedam, direta ou indiretamente, conhecimento e capital social para seus pares (GIMENEZ; TACHIZAWA, 2012; BRUNEEL; HELENA; YLI, 2014) e assim possam melhorar o desempenho em áreas como design de produto, marketing, logística, pesquisa e desenvolvimento, sourcing, e serviço ao cliente (PARK; SUBRAMANIAM; STYLIANOU, 2015).

O Quadro 2 apresenta as principais formas de aprendizagem que podem ser adotadas por empresas de um APL.

Quadro 2 - Formas de aprendizado

\begin{tabular}{|c|c|c|}
\hline Autor & $\begin{array}{c}\text { Formas de } \\
\text { Aprendizagem }\end{array}$ & Esclarecimentos \\
\hline Ludvall (1988). & $\begin{array}{l}\text { Learning-by- } \\
\text { interacting ou } \\
\text { learning-by- } \\
\text { cooperating }\end{array}$ & $\begin{array}{l}\text { O aprendizado ocorre através de um } \\
\text { organizado e continuo fluxo de informações } \\
\text { e canais de comunicação entre diferentes } \\
\text { agentes (econômicos, institucionais e } \\
\text { governamentais), que cooperam } \\
\text { disponibilizando tempo, confiança e } \\
\text { investimento necessários para o } \\
\text { compartilhamento e assimilação de } \\
\text { conhecimentos entre organizações, assim } \\
\text { como para o retorno futuro de benefícios } \\
\text { financeiros. }\end{array}$ \\
\hline \multirow{2}{*}{$\begin{array}{l}\text { Panjaitan e } \\
\text { Noorderhaven (2008) }\end{array}$} & Aprendizado formal & Intercâmbio formalizado de conhecimentos. \\
\hline & Aprendizado informal & $\begin{array}{l}\text { Transmissão de conhecimento tácito pela } \\
\text { interação e partilha espontânea de } \\
\text { informações. }\end{array}$ \\
\hline \multirow[t]{2}{*}{$\begin{array}{l}\text { Bruneel, Helena e Yli } \\
\text { (2014), }\end{array}$} & Modelagem & $\begin{array}{l}\text { Observação e imitação de práticas de outras } \\
\text { organizações, sem necessariamente } \\
\text { cooperarem entre si. }\end{array}$ \\
\hline & Transferência & $\begin{array}{l}\text { Transferência formal de conhecimentos em } \\
\text { relações estabelecidas de cooperação. }\end{array}$ \\
\hline \multirow[t]{2}{*}{$\begin{array}{l}\text { Gibb, Sune e Albers } \\
(2016)\end{array}$} & Aprender a competir & $\begin{array}{l}\text { Aprendizagem em rede, onde pequenas firmas } \\
\text { colaboram e aprendem como um grupo para } \\
\text { resolver problemas e alcançar metas de } \\
\text { desempenho individual. }\end{array}$ \\
\hline & Aprender a executar & $\begin{array}{l}\text { Aprendizagem que visa maximizar a } \\
\text { transferência e adoção de conhecimento do } \\
\text { grupo como um todo, para otimizar o } \\
\text { desempenho coletivo. }\end{array}$ \\
\hline
\end{tabular}

Fonte: Elaborado pelos autores (2018)

Zambrana e Teixeira (2015) complementam que para que o aprendizado interorganizacional em APLs seja mais profícuo é relevante a presença e interação entre diferentes agentes econômicos, políticos, institucionais e suas respectivas contribuições. Além disso, é necessário averiguar se as empresas parceiras REUNA, Bela Horizonte - MG, Brasil, v.24, n.3, p. 20-40, Jul. - Set. 20IG - ISSN 2179-8834 
compartilham de lógicas institucionais e percepções empresariais semelhantes, assim como se estão numa posição de equilíbrio entre colaboração e competição (MELO; BECK, 2015), uma vez que a aprendizagem interorganizacional está diretamente relacionada a postura empresarial (gestor) adotada por cada organização (VASUDEVA; ALEXANDER; JONES, 2015). Por essa razão Sie et al., (2014) reforçam o entendimento de que o aprendizado bem sucedido depende de decidir correta e estrategicamente com quem cooperar.

Conforme foi apresentado, anteriormente, a aprendizagem interorganizacional é expressivamente constituída pela comunicação e interação entre membros de organizações cooperantes. Nesse contexto desponta o papel das tecnologias de informação e comunicação, com suas diversas funcionalidades que facilitam e promovem o compartilhamento de informações e conhecimentos no nível individual, organizacional e interorganizacional (PARK; SUBRAMANIAM; STYLIANOU, 2015).

Para o aprendizado interorganizacional, o papel da TI envolve a geração e compartilhamento de conhecimentos explícito e tácito em cadeias virtuais de comunicação, que conectam eletronicamente organizações parceiras (SCOTT, 2000) por meio de redes sociais digitais (SANCHES, MUINA, 2011).

As redes sociais digitais (RSD), também chamadas de redes sociais online ou redes sociais virtuais (KATONA; ZUBCSEK; SARVARY, 2011) são, no ambiente da internet, teias de relacionamentos formadas em canais da web ou aplicativos móveis, que proporcionam a interação e rápida troca de informações entre membros individuais e/ou organizacionais de comunidades online (ALMÉRI et al., 2013; NEGREIROS, 2015).

No Brasil, de acordo com o We are Social (DIGITAL IN 2016) o Facebook, o WhatsApp, o Messenger, o Youtube e o Instagram, são, as redes sociais digitais mais utilizadas.

No Brasil, de acordo com o We are Social (DIGITAL IN 2016) o Facebook, o WhatsApp, o Messenger, o Youtube e o Instagram são as redes sociais digitais mais utilizadas. Dada a facilidade de utilização, baixo custo e disponibilidade, essas redes foram consideradas com amplo potencial para comunicação, cooperação e aprendizado interorganizacional em APLs.

É de ressaltar que essas redes sociais digitais, aliadas a e-mails, ferramentas do Google Group e etc., têm sido utilizadas como fontes de armazenamento de informações eletronicamente acessíveis por funcionários de empresas parceiras que compartilham conhecimentos e experiências para a resolução de problemas, tomada de decisão e aprendizado interorganizacional (PARK; SUBRAMANIAM; STYLIANOU, 2015). 


\section{Procedimentos metodológicos}

Conforme Fontelles et al., (2009) existem diversas formas de classificar uma pesquisa, e os autores não são unânimes quanto à padronização desta classificação. Por essa razão, essas autoras definiram que as pesquisas podem ser classificadas, dentre outras formas, quanto à: forma de abordagem, objetivos, procedimentos técnicos e desenvolvimento no tempo.

Quanto a forma de abordagem esse estudo pode ser classificado como uma pesquisa qualitativa por buscar compreender o fenômeno da cooperação e aprendizado no APL de TI de Aracaju/SE a partir da interpretação dos participantes e no contexto em que ocorre tal fenômeno (LEÃO, MELO, VIEIRA, 2009). No tocante aos objetivos, esse estudo é caracterizado como descritivo por buscar descrever e compreender como ocorre o fenômeno estudado e quem são os atores participantes (NEUMAN, 1997)

No que concerne aos procedimentos técnicos foi utilizada a pesquisa bibliográfica e a pesquisa de campo, com estratégia do tipo estudo de casos múltiplos. A estratégia de estudo de caso foi adotada por permitir a compreensão das dinâmicas de cooperação e aprendizagem presentes dentro do APL de TI de Aracaju/SE (EISENHARDT, 1997). O método de estudo de casos múltiplos foi preferido por contemplar o estudo de caso único do arranjo produtivo local de $\mathrm{TI}$ e de suas diferentes organizações como múltiplas unidades de análise (YIN, 2015). As unidades de análise foram selecionadas por critérios de acessibilidade dentre uma lista de empresas sugeridas pelo órgão gestor de APLs em Aracaju/SE - SEDETEC/SE.

Para coleta dos dados, foi utilizado um roteiro de entrevista semiestruturada construído a partir da revisão da literatura e fundamentado nas seguintes categorias analíticas: características do empreendimento; cooperação e aprendizado interorganizacional e redes sociais digitais. As entrevistas foram realizadas com sócios-gestores de três empresas participantes do APL de TI de Aracaju/SE no período compreendido entre 24 de setembro e 17 de outubro de 2018.

A análise dos dados foi feita por meio de uma análise descritiva qualitativa dos resultados identificados. Nessa análise realizou-se a cross-case analysis, pela qual buscou-se identificar diferenças e aspectos comuns entre as unidades de análise consideradas, assim como foram confrontados resultados obtidos com os postulados teóricos consultados (EISENHARDT, 1989).

\section{Apresentação e análise dos casos}

O caso selecionado para a pesquisa foi o do Arranjo Produtivo Local de Tecnologia da informação de Aracaju/SE. Esse arranjo teve início em 1990 quando se formaram no estado de Aracaju/SE as primeiras empresas de TI. O APL de TI de Aracaju/SE é formado por 98 empresas, sendo a maioria desses empreendimentos classificados como micro e pequenos negócios. Essas empresas estão, majoritariamente, localizadas na capital Aracaju, e em outras 10 cidades do estado (MTE/RAIS, 2015).

Os principais produtos ofertados pelas empresas participantes do APL de TI de Aracaju/SE contemplam: Consultoria em Tecnologia da Informação; Desenvolvimento de programas de computador sob encomenda; Suporte técnico, manutenção e outros 
serviços em tecnologia da informação; e Tratamento de dados, provedores de serviços de aplicação e serviços de hospedagem na internet.

As múltiplas unidades de análise desse estudo foram constituídas por três empresas de TI que atuam em Aracaju, as quais serão identificadas como empresas A, B e C. As principais características dessas empresas estão representadas no Quadro 3.

Quadro 3 - Unidades de análise

\begin{tabular}{|c|c|c|c|}
\hline Empresa & $\mathbf{A}$ & B & C \\
\hline № funcionários & 3 & 5 & 5 \\
\hline Tamanho & Microempresa & Microempresa & Microempresa \\
\hline Fundação & 2012 & 2003 & 2002 \\
\hline Entrevistado & Sócio-gestor & Sócio-gestor & Sócio-gestor \\
\hline Produtos & $\begin{array}{l}\text { Soluções Web: } \\
\text { Responsive web; } \\
\text { Design; } \\
\text { HTML5, } \\
\text { Aplicativos mobile. }\end{array}$ & $\begin{array}{l}\text { Sistemas de gestão: } \\
\text { Folha de pagamentos; } \\
\text { Contábil; } \\
\text { Patrimonial; } \\
\text { Livros fiscais; } \\
\text { Controle para } \\
\text { almoxarifados } \\
\text { Gestão parlamentar } \\
\text { web; } \\
\text { ERP de gestão web } \\
\text { empresarial. }\end{array}$ & $\begin{array}{l}\text { Soluções em } \\
\text { automação } \\
\text { comercial para de } \\
\text { postos de } \\
\text { combustíveis } \\
\text { Suprimentos de TI. }\end{array}$ \\
\hline Atuação & Internacional & Nacional & Regional \\
\hline
\end{tabular}

Fonte: Elaborado pelos autores.

Dada a constante interconexão entre os temas cooperação, aprendizado e redes sociais digitais, nas empresas pesquisadas, essas categorias de análise foram tratadas em tópico único, conforme segue.

\section{Cooperação e aprendizado pelo uso de redes sociais digitais no APL de tecnologia da informação de Aracaju/SE}

Inicialmente, foi questionado as organizações participantes o que entendiam por cooperação interorganizacional. Os entrevistados declararam que:

\footnotetext{
"Veja, nós buscamos manter um contato e bom relacionamento com as outras empresas de $\mathrm{TI}$, principalmente startups, isso é importante para fortalecer nosso setor e acompanhar o que o pessoal está fazendo. Mas cooperação e parceria mesmo não firmamos com outras empresas não." (ENTREVISTADO EMPRESA A, 2018).
}

As empresas $B$ e $C$ corroboram a percepção da empresa $A$, mas destacaram a existência de interações em níveis mínimos, conforme é percebido na fala desses entrevistados: 
"Não existe uma cooperação em grandes termos, principalmente porque não são as empresas que cooperam entre si, mas sim uma boa relação entre os proprietários e desenvolvedores dessas empresas que já se conhecem há muitos anos e mantêm uma relação harmoniosa e cordial entre si e sempre que possível auxiliam o amigo, mas cada um com o seu negócio." (EMPRESA B, 2018). destacou que:

O entrevistado da empresa $\mathrm{C}$ complementou as percepções da empresa $\mathrm{B}$ e "Todas as empresas de automação e desenvolvimento de softwares
são geridas por pessoas que em algum momento da vida trabalharam
juntos, seja dando aula ou participando de cursos específicos, são
vínculos de amizade que permitem comunicação e uma pontinha de
cooperação" (ENTREVISTADO EMPRESA C, 2018).

Conforme pôde ser percebido na fala dos entrevistados, a cooperação entre as empresas do APL de TI da grande Aracaju ainda se encontra em níveis incipientes, que não ultrapassaram barreiras de comunicação informal entre seus gestores. Brass et al.,(2004) destacam que a colaboração entre organizações passa por três níveis: (1) quando indivíduos que fazem parte de grupos dentro de diferentes organizações interagem entre si; (2) quando grupos/departamentos que fazem parte de organizações interagem com outros grupos de outras empresas e, por fim, (3) quando as organizações, em termos de gestão e cultura estratégica, fazem parte formal e incisiva em redes de cooperação. Conforme pôde ser percebido nos casos relatados, no APL de TI de Aracaju/SE a cooperação encontra-se num estágio embrionário, pelo qual os indivíduos colaboram entre si, numa informal troca de informações, mas não há efetiva cooperação entre as organizações.

A cooperação informal, assim como acontece entre as empresas pesquisadas é mais comum que a cooperação formal, principalmente em micro e pequenas empresas que estabelecem vínculos informais para realização de atividades de pequeno impacto para seus negócios (GEROLÁMO et al., 2008). Conforme pôde ser percebido a cooperação entre as organizações estudadas surgiu por vínculos sociais dos membros dessas empresas, vínculos estes que já existiam antes mesmo da constituição desses negócios. Essa percepção é ratificada por Gonçalves (2014) que evidenciou que a cooperação informal surge a partir das ações de indivíduos, e não necessariamente de organizações, que mantém vínculos sociais e de convivência, além das relações empresariais.

É relevante destacar, e também em consequência do aspecto informal da cooperação detectada, que essa, segundo todos os relatos, não foi fomentada por políticas governamentais e que surgiu espontaneamente entre os empresários, sendo inclusive consenso entre os entrevistados que não existem políticas públicas claras ou devidamente divulgadas que incentivem a cooperação interorganizacional no estado de Aracaju/SE.

Foi percebido que as relações de cooperação entre as empresas pesquisadas no APL em estudo são constituídas, prioritariamente, pela troca de informações, e conforme relato dos entrevistados essa interação não acontece unicamente por meios presenciais, mas também e frequentemente por meio de redes sociais digitais, a partir das quais, também, são desenhadas práticas de cooperação. 
O entrevistado da empresa A relatou, por exemplo, que faz amplo uso da rede social Facebook. A empresa participa de uma comunidade virtual na qual diferentes empresas interagem entre si divulgando inovações e sanando dúvidas técnicas. O entrevistado relatou que:

\begin{abstract}
"Nos temos uma comunidade no Facebook, a intenção da comunidade é fortalecer as empresas com potencial inovador do estado e a gente se ajudar lá. Acontece, frequentemente, de várias empresas utilizarem uma mesma plataforma para desenvolvimento de software e então compartilhamos e trocamos informações sobre os melhores plugins, criptografia, códigos e sistemas para melhor utilização dessas plataformas. Basta que alguém faça a publicação para alcançar no mínimo 20 comentários" (EMPRESA A, 2018).
\end{abstract}

Segundo o entrevistado, o Facebook é a ferramenta mais adequada para comunicação entre as empresas, porque em maior ou menor frequência quase todos os indivíduos se conectam a rede. Além disso, pelo Facebook, segundo a empresa A, o compartilhamento de informações é mais fácil e tem alcance para todos os participantes do grupo. Essa evidência foi constatada pelo postulado de Gunelius (2012) que aponta a vantagem do Facebook em permitir a criação de relacionamentos, de livre ou restrito acesso, nos quais indivíduos, instituições e/ou organizações podem debater temas comuns e compartilhar conteúdos entre si.

As empresas $B$ e $C$ fazem parte de um grupo WhatsApp. Conforme relatos do entrevistado da empresa $B$, corroborados na descrição da empresa $C$, esse grupo foi criado em meados de 2013 como um meio de promover maior interação entre as empresas de TI do estado.

Os entrevistados destacaram que o uso do WhatsApp é justificado, simplesmente, por ser uma ferramenta que "todo mundo" tem acesso, o uso é gratuito e a comunicação rápida. Essa constatação pode ser validada pelo entendimento de Ferreira e Arruda Filho (2016) que reforçam que o sucesso do WhatsApp está atrelado ao potencial de interação, velocidade na comunicação, sociabilização e troca de mensagens entre usuários, que tornam o aplicativo, cada vez mais, uma ferramenta de trabalho para os mais diversos segmentos. Além do WhatsApp essas empresas utilizam também o Google Groups e Telegram, mas segundo os entrevistados essas ferramentas são raramente acionadas.

No tocante a forma como a cooperação ocorre por meio do WhatsApp, o entrevistado da empresa B destacou que:

"A gente coopera tirando dúvidas com nossos parceiros, como isso acontece?! Quando uma empresa tem alguma dúvida relacionada a legislação ou como resolver algum problema de desenvolvimento ou automação já pergunta logo as outras empresas do grupo. Por exemplo, recentemente houve uma mudança na forma de emissão de notas fiscais eletrônicas então vários colegas postaram a dúvida no grupo e cada empresa deu sua colaboração sobre como se ajustar a mudança. Se você olhar o histórico (do grupo) vai perceber que todos os dias novas informações são compartilhadas e são informações úteis para os nossos negócios" (ENTREVISTADO EMPRESA B, 2018).

RELUNA, Belo Horizonte - MG, Brasil, v.24, n.3, p. 20-40, Jul. - Set. 2019 - ISSN 2179-8834 
É importante destacar que os relatos do entrevistado na empresa $C$ seguem na mesma direção do apresentado pela empresa B. Contudo, aquele entrevistado acrescentou que:

"Nós usamos o grupo mesmo para nos atualizarmos sobre mudanças na legislação trabalhista e trocar ideias sobre novas tecnologias. Mas acontece também, com muita raridade, de alguém pedir algum equipamento emprestado, eu mesmo já pedi e emprestei algumas vezes. É mais fácil colocar no grupo porque todos visualizam e não é preciso ficar ligando de um em um. Ah, esse empréstimo é geralmente muito rápido, porque cada empresa tem grande necessidade dos seus equipamentos" (ENTREVISTADO EMPRESA C, 2018.).

Quando questionados sobre aprendizado interorganizacional, os entrevistados relataram que: "Querendo ou não nos estamos criando e compartilhando conhecimentos e pelo o que eu vejo lá na página esse conhecimento tem sido utilizado e é relevante para as empresas" (ENTREVISTADO EMPRESA A, 2018).

O entrevistado B, por sua vez, disse que:

"Na medida que eu tenho acesso a qualquer informação dos concorrentes, porque somos antes de tudo concorrentes, eu estou tornando minha empresa mais dinâmica e competitiva porque estou incrementando meu conhecimento interno e sabendo que os males que me afligem também atingem as outras empresas" (ENTREVISTADO EMPRESA B, 2018).

Esse entrevistado ainda completa que:

"Olha foi a partir de discussões no grupo que eu conheci novas funcionalidades da impressão remota através do google print e de desenvolvimento mobile, então não tenho como dizer que meu negócio não foi beneficiado ou não aprendeu coisas novas" (ENTREVISTADO EMPRESA B, 2018).

O entrevistado da empresa $\mathrm{C}$ relata que:

"Olha eu tenho batido na tecla que é preciso formalizar essa cooperação, porque eu sinto aquele grupo como uma extensão de nossa amizade, a gente troca informações e compartilha novidades, mas nenhum processo dentro da minha empresa foi expressivamente melhorado por causa do grupo não. Aliás, na internet, participo de grupos e fóruns com empresas de todo o mundo, com robustos bancos de dados que eu aprendo e implemento muito mais" (ENTREVISTADO EMPRESA C, 2018).

Conforme pôde ser percebido a partir dos relatos, o aprendizado entre as empresas de TI do APL de Aracaju/SE constitui-se, prioritariamente, pelo compartilhamento de conhecimentos tácitos. Essa evidência é validada por Mendes Filho (2009) que destacou que nos APLs o aprendizado é mais expressivo pelo compartilhamento informal do conhecimento tácito integrado em indivíduos, organizações e na própria localidade. Essa evidência ainda vai de encontro a REUNA, Belo Horizonte - MG, Brasil, v.24, n.3, p. 20-40, Jul. - Set. 2019 - ISSN 2179-8834 
Panjaitan e Noorderhaven (2009) que defende que o processo de aprendizagem não pode ser restringido, apenas, a uma troca de documentos entre portadores formais de informação. Em vez disso, as interações sociais entre membros individuais das organizações colaboradoras devem ser intensas e estreitas o suficiente para permitir o intercâmbio e transferência do componente tácito do conhecimento, conforme foi percebido no caso analisado.

A partir das colocações dos entrevistados foi possível perceber que para as empresas $\mathrm{A}$ e $\mathrm{B}$, ainda que sem assumir um caráter formal, os conhecimentos colhidos a partir da interação pelo uso das RSD promovem um aprendizado que direta e/ou indiretamente são utilizados nos processos produtivos dessas organizações. Para a empresa C, por sua vez, o aprendizado interorganizacional está restrito, prioritariamente, a troca de informações sobre legislação trabalhista e, em menor grau, sobre os processos produtivos de cada organização.

Os relatos dos entrevistados sobre o aprendizado promovido pela cooperação vão de encontro aos postulados teóricos que apontam que, conforme ocorre nas empresas $A$ e $B$, principalmente, no processo de aprendizagem interorganizacional uma firma interpreta, transforma e aloja novos conhecimentos sobre produtos e processos tecnológicos, advindos de outras organizações, e, em seguida, emprega estes conhecimentos para melhorar suas atividades organizacionais (YANG et al., 2014).

Mesmo tendo sido destacado por todas as organizações entrevistadas a importância das redes sociais digitais, foi unanime o entendimento, entre os participantes desse estudo, que ainda que promova facilidades para comunicação, interação e cooperação, as redes sociais digitais não substituem o contato e interação pessoal entre indivíduos, mas facilitam e dinamizam os encontros presenciais.

É de ressaltar que embora os relatos apontem a importância das informações obtidas de outras organizações, essas informações não foram em nenhuma das unidades de análise apontadas como expressivas de fontes de aprendizado para essas empresas. Esse fato merece destaque porque os gestores entrevistados indicaram que a sua principal fonte de conhecimentos internamente era o processo produtivo, e externamente as demandas e exigências dos clientes. De acordo com esses gestores, as exigências dos clientes obriga a empresa a buscar novos conhecimentos que, em maior ou menor grau são utilizados no processo produtivo, sendo por essa razão o processo produtivo indicado como principal fonte de conhecimento e aprendizado pela totalidade das empresas pesquisadas.

Nenhum dos entrevistados julgou como expressivo e substancial o conhecimento adquirido pela interação com outras organizações, contudo indicaram que as informações e conhecimentos assimilados pelo uso das RSD são, indiretamente, empregados em seus processos produtivos, o que demonstra que ainda que os empreendedores não tenham se atentado, a cooperação e o aprendizado interorganizacional têm influenciado no funcionamento de seus respectivos negócios. 
Os resultados apresentados para o APL em estudo estão no Quadro 4.

Quadro 4 - Resumo dos resultados

\begin{tabular}{|c|c|c|c|}
\hline Empresa & A & B & C \\
\hline \multirow{3}{*}{$\begin{array}{l}\text { Redes Sociais } \\
\text { utilizadas }\end{array}$} & \multirow[t]{3}{*}{ Facebook } & WhatsApp & WhatsApp \\
\hline & & Google Groups & \multirow[t]{2}{*}{ Google Groups } \\
\hline & & Telegram & \\
\hline \multirow{6}{*}{$\begin{array}{l}\text { Cooperação } \\
\text { (Compartilhamento de } \\
\text { Informações) }\end{array}$} & $\begin{array}{l}\text { Desenvolvimento de } \\
\text { software }\end{array}$ & Legislação trabalhista & $\begin{array}{l}\text { Legislação } \\
\text { Trabalhista }\end{array}$ \\
\hline & Plugins & Google Print & $\begin{array}{l}\text { Empréstimo de } \\
\text { equipamentos }\end{array}$ \\
\hline & Criptografia & $\begin{array}{l}\text { Desenvolvimento } \\
\text { mobile }\end{array}$ & \\
\hline & $\begin{array}{l}\text { Códigos para } \\
\text { utilização de } \\
\text { plataformas }\end{array}$ & & \\
\hline & $\begin{array}{l}\text { Desenvolvimento } \\
\text { mobile }\end{array}$ & ----------------------- & ---------------------- \\
\hline & & $\begin{array}{l}\text { Incremento aos } \\
\text { processos produtivos }\end{array}$ & \\
\hline Vínculos & Informal & Informal & Informal \\
\hline Aprendizado & $\begin{array}{l}\text { Incremento aos } \\
\text { processos produtivos }\end{array}$ & $\begin{array}{l}\text { Atualização quanto a } \\
\text { mudanças na } \\
\text { legislação. }\end{array}$ & $\begin{array}{l}\text { Atualização quanto } \\
\text { a mudanças na } \\
\text { legislação. }\end{array}$ \\
\hline
\end{tabular}

Fonte: Elaborado pelos autores a partir da pesquisa de campo.

Dentre as empresas pesquisadas que compõem o APL de TI de Aracaju/SE foi verificado que a cooperação interorganizacional encontra-se num estágio embrionário e com atividades incipientes de cooperação entre as organizações. A partir dos relatos dos entrevistados foi perceptível que existe uma intenção latente de formalizar as atividades de cooperação, contudo, essa intenção tem sido renegada em razão da limitação temporal dos empreendedores, carecendo, assim, de orientação mais incisiva de algum órgão patronal ou política pública para que a cooperação entre essas empresas alcance novos patamares e promova melhores resultados operacionais, organizacionais e estratégicos às empresas envolvidas.

A partir dos níveis incipientes de cooperação entre as empresas pesquisadas, e considerando o potencial dessas instituições para formação de parcerias propõe-se, a seguir, um modelo teórico para cooperação e aprendizado pelo uso de redes sociais digitais no APL de tecnologia da Informação de Aracaju/SE.

\section{Proposta de modelo de aprendizagem interorganizacional utilizando redes sociais digitais}

Inicialmente, é de ressaltar que o modelo proposto considera empresas de micro e pequeno porte, envolvidas em atividades correlatas, e por essa razão adota a estrutura de cooperação horizontal. O modelo proposto considera que a cooperação interorganizacional é precedida pela cooperação individual entre membros 
participantes de diferentes organizações que mantém algum nível de comunicação e interação entre si (BRASS et al., 2004). A partir dessa cooperação individual, as organizações percebem a existência de objetivos e dificuldades em comum, consideram a atividade cooperada e selecionam possíveis parceiros. A escolha dos possíveis aliados considera o capital social e a predisposição à cooperação demonstrada por essas empresas.

Vencida a fase de seleção de parceiros as organizações definem, em razão de seus objetivos, quais as atividades que devem ser cooperadas com outras organizações. Para o setor de tecnologias de informação sugeriu-se ações conjuntas para desenvolvimento de softwares, produtos inovadores, prospecção de mercados, compras conjuntas, qualificação da produção, divisão de serviços, compartilhamento de recursos financeiros e materiais, e prestação de serviços em consultoria especializada. Explica-se que para essa última ação a intenção é que as organizações parceiras, cada qual com suas especialidades e competências ofereçam suporte em consultoria a outras de empresas de $\mathrm{TI}$, assim como organizações de quaisquer outros setores.

Conforme é exposto no modelo após a definição das atividades a serem operadas por cooperação, as organizações envolvidas decidem se adotam relações formais ou informais na parceria. As relações formais serão guiadas por trâmites contratuais, ao passo em que as relações informações serão pautadas na confiança, convivência e amizade entre os membros da equipe de cooperação (BORTOLASO; VERSCHOORE; VALE JR, 2012).

Figura 1: Proposta de modelo teórico para cooperação e aprendizado pelo uso de redes sociais digitais 


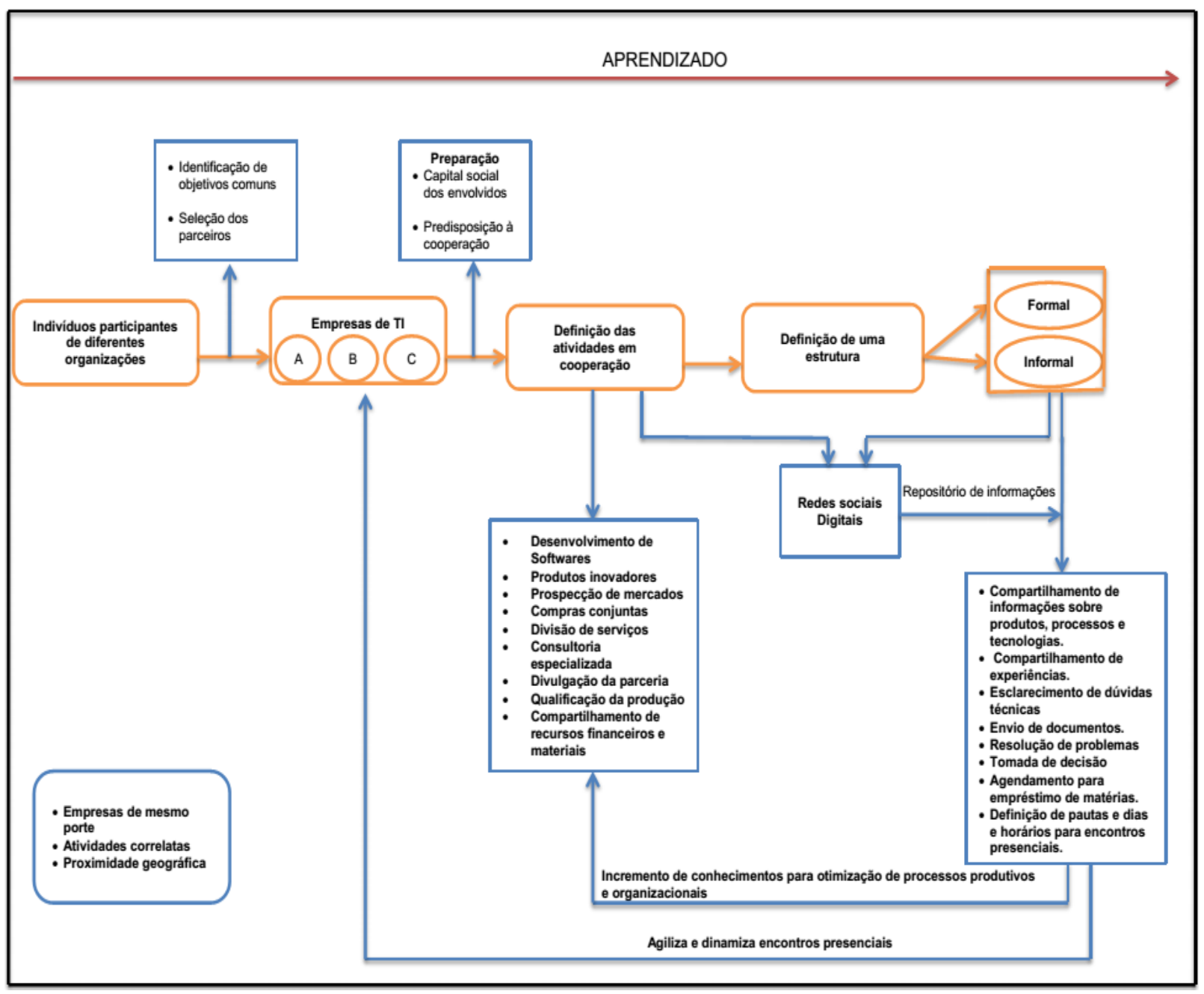

Fonte: Elaborado pelos autores (2018)

Independente da formalidade dos acordos de parceria, esses poderão utilizarse das redes sociais digitais para promover a cooperação e, simultaneamente, disponibilizar/assimilar conhecimentos para as empresas envolvidas. A ideia subjacente é que por meio das interações usando redes sociais digitais a exemplo do: Facebook, WhatsApp, Messenger, Google Groups, e-mail, Skype, Instagram, dentre outros - as organizações distribuam conhecimentos sobre produtos e processos tecnológicos, assim como conversem e esclareçam dúvidas técnicas, agilizem a tomada de decisão em menor tempo (em função das informações disponibilizadas), organizem listas para empréstimos de equipamentos, compartilhem documentos entre si e até mesmo definam pautas, datas e horários para encontros presenciais.

Mais que isso, o modelo propõe que as redes sociais digitais possam ser utilizadas como repositórios de informações os quais poderão, constantemente, ser consultados e assim promover aprendizado interorganizacional capaz de otimizar os processos produtivos e organizacionais das empresas envolvidas em razão do conhecimento acumulado.

Assim, o modelo proposto demonstra que desde a etapa de interação individual até a colaboração por meios virtuais, o aprendizado está presente em todas as etapas da cooperação, isso porque as empresas participantes estão em constante processo 
de buscar informações sobre parceiros, processos produtivos, atividades cooperadas e resultados possíveis. Toda essa informação gera conhecimentos de caráter tácito e explícito, que associados às atividades de cooperação, e ao uso das redes sociais digitais como repositório de informações e conhecimentos, promovem condições para sobrevivência, vantagem competitiva e destaque empresarial para as empresas participantes.

\section{Conclusões}

Este artigo teve como principal objetivo analisar como o uso de redes sociais virtuais tem colaborado para praticas cooperativas e aprendizado no arranjo produtivo local de Tecnologia da informação em Aracaju/SE. Notabilizou-se que os arranjos produtivos locais tendem a promover cooperação empresarial entre organizações, principalmente organizações de micro e pequeno porte que geralmente enfrentam desafios semelhantes para se manter no mercado, e que devido a proximidade geográfica acabam interagindo e promovendo atividades cooperadas formais e informais, com diferentes níveis de integração interorganizacional, em prol de maior aprendizado, acesso a recursos e alcance de objetivos similares.

Para melhor averiguar a conexão entre os temas cooperação, aprendizado e redes sociais virtuais, essa pesquisa realizou um estudo de casos múltiplos no arranjo produtivo local de tecnologia da informação de Aracaju/SE. Os resultados apontam que as empresas pertencentes ao APL de TI de Aracaju/SE promovem uma cooperação baseada prioritariamente no compartilhamento de informações, mas que não se constituem na operacionalização de atividades conjuntas propriamente ditas.

O estudo revelou ainda que a incipiente cooperação entre as empresas do APL estudado é facilitada pelo uso de redes sociais digitais, prioritariamente do WhatsApp e Facebook, assim como do Telegram e Google Groups. Conforme foi percebido as empresas utilizam essas ferramentas para compartilhar conhecimentos técnicos, trocar experiências e se atualizar quanto a mudanças em legislação especifica. Destaca-se também que o uso das redes sociais digitais promove um aprendizado interorganizacional baseado no compartilhamento de conhecimentos tácitos que são interpretados e aplicados, conforme percepção dos gestores, nos processos produtivos e organizacionais das organizações participantes do APL.

Constatada a embrionária cooperação entre as empresas do APL de TI de Aracaju/SE, esse estudo propôs um modelo conceitual para cooperação e aprendizado pelo uso de redes sociais digitais em empresas de TI. A intenção do modelo proposto foi demonstrar que a maior utilização de redes sociais digitais nas atividades de cooperação cria condições para outras formas de cooperação e aprendizado que melhoram as condições competitivas das firmas envolvidas. É de ressaltar que o modelo proposto não intenciona substituir o contato e interação humana, e sim dinamizar a comunicação, cooperação e aprendizado entre empresas envolvidas em atividades colaborativas.

Assim, conforme pesquisa bibliográfica e evidências da pesquisa de campo, percebeu-se que as atividades cooperadas têm potencial para promover expressivos ganhos econômicos, acesso a recursos e conhecimentos, e assim alavancar a credibilidade empresarial para organizações participantes, contudo, é necessários que essas organizações adotem posturas e estratégias mais incisivas sobre 0 
comportamento cooperativo e uso de RSD, caso contrários os resultados serão parciais e de amplitude limitada.

Dentre as principais limitações nesse estudo destaca-se inicialmente que por ser um estudo de casos os resultados não podem ser generalizados, sendo diretamente relacionados ao APL e empresas estudadas. Além disso, o reduzido número de organizações pesquisadas pode ter minorado os efeitos da realidade de cooperação, aprendizado e uso de redes sociais digitais entre as empresas participantes do APL.

Recomendam-se novos estudos com a participação de um maior número de organizações para contrastar com os resultados aqui apresentados, assim como sugere-se, também, a replicação dessa pesquisa incluindo atores institucionais e políticos que interagem no APL, com a intenção de verificar como esses atores visualizam a cooperação e o aprendizado e se também fazem uso de redes sociais digitais. Outra sugestão de pesquisa é analisar porque, de acordo com a percepção dos entrevistados, os órgãos gestores de APL em Aracaju/SE não promovem, de modo mais incisivo, cooperação entre as empresas, contrastando assim percepções governamentais e empresariais.

\section{Referências}

BALESTRIN, A.; VERSCHOORE, J. R.; REYES Jr., E. O campo de estudo sobre redes de cooperação interorganizacional no Brasil. Revista de Administração Contemporânea, [S. I.], v. 14, n. 3, p. 459-477, 2010.

BORTOLASO, I. V.; VERSCHOORE, J. R.; VALE JR, Á. S. E. do. O relacionamento sustenta a cooperação empresarial? Uma análise do relacionamento interno em duas redes horizontais. Análise, Porto Alegre, v. 23, n. 3, p. 234-243, set.-dez. 2012.

BRASS, D.; GALASKIEWICZ, J.; GREVE, H.; TSAI, W. Taking Stock of Networks and Organizations: A Multilevel Perspective. Academy of Management Journal v. 47(6), p. 795-817, 2004.

BRUNEEL, J.; R.; HELENA; YLI; B., Clarysse. Learning from experience and learning from others: how congenital and interorganizational learning substitute for experiential learning in young firm internationalization. Strategic Entrepreneurship Journal Strat. Entrepreneurship J., v. 4, p. 164-182, 2010.

CASSIOLATO, J. E.; LASTRES, H. M. M.; MACIEL M. L. (orgs.) Pequena Empresa: cooperação e desenvolvimento local. Rio de Janeiro: Relume Dumará, 2003.

CASTRO, L. H. Arranjo produtivo local. Brasília: SEBRAE, 2009. 44 p. (Série Empreendimentos Coletivos).

COGHLAN, D.; COUGHLAN, P. Effecting Change and Learning in Networks through Network Action Learning. The Journal of Applied Behavioral Science, v. 51 (3) 375400, 2015. 
COLET, D. S.; Importância da aprendizagem interorganizacional para micro e pequenas empresas: proposição de um framework de análise. In: IX Encontro de estudos sobre empreendedorismo e gestão de pequenas empresas, 2016, Passo Fundo. Anais... Passo Fundo: Universidade de Passo Fundo, 2016.

EISENHARDT, K. M. Building theories from case study research. Academy of Management, v. 14, n.4, p. 532-550, Oct. 1989.

ESTEVES, Gina Maria da Rocha. Cooperação Empresarial: Proposta de um Modelo para o Setor dos Transportes/Logística. 2012, 93 f. Dissertação (Mestrado em ciencias sociais e humanas) - Universidade da Beira Interior, Covilhã, Portugal, 2012.

FERREIRA, N. S.; ARRUDA FILHO, J. M. Facebook e WhatsApp: Uma análise das preferências de uso. REUNA, Belo Horizonte - MG, v.20, n.3, p. 47-64, Jul. - Set. 2016.

FONTELLES, M. J.; SIMÕES, M. G.; FARIAS, S. Hasegawa; FONTELLES, R. G. S. Metodologia da pesquisa científica: Diretrizes para a elaboração de um protocolo de pesquisa. Trabalho realizado no Núcleo de Bioestatística Aplicado à pesquisa da Universidade da Amazônia - UNAMA, 2009.

GEROLÁMO, M. C.; CARPINETTI; L. C. R.; FLESCHUTZ, T.; SELIGER, G. Clusters e redes de cooperação de pequenas e médias empresas de PMEs: observatório europeu, caso alemão e contribuições ao caso brasileiro. Gestão da Produção, São Carlos, v. 15, n. 2, p. 351-365, maio-ago. 2008.

GIBB, J.; SUNE, A.; ALBERS, S. Network learning: Episodes of interorganizational learning towards a collective performance goal. European Management Journal xxx, p. 1e11, 2016.

GONÇALVES, A. C. B.. A cooperação informal para a internacionalização. 2014, 110 f. Dissertação (Mestrado em Gestão e Internacionalização de Empresas) - IPP Escola superior de tecnologia e Gestão, Felgueiras, Portugal, 2014.

GOVERNO DE SERGIPE. Secretaria de Estado do Desenvolvimento Econômico e da Ciência e Tecnologia. Plano de desenvolvimento do arranjo produtivo de tecnologia da informação da grande Aracaju, 2009.

GUNELIUS, S. Blogging All-in-One For Dummies. 2012.

KATONA, Z.; ZUBCSEK, P. P.; SARVARY, M. Network Effects and Personal Influences: The Diffusion of an Online Social Network. Journal of Marketing Research,vol. 48, n. 3, p. 425-443, june, 2011.

LEÃO, A. L. M. S.; MELLO, S. B. C.; VIEIRA, R. S. G. O papel da teoria no método de pesquisa em Administração. Revista Organizações em Contexto, v. 5, n. 10, p. 116, 2009.

MARTINS, D. M.; FARIA, A. C. De; FARINA, M. C. Cooperação e poder na qualidade do relacionamento das cooperativas de crédito. $R$. Adm. FACES Journal Belo Horizonte, v. 15 n. 2 p. 25-45 abr./jun. 2016. 
MELO, S.; BECK, M. Intra and Interorganizational Learning Networks and the Implementation of Quality Improvement Initiatives: The Case of a Portuguese Teaching Hospital. Human resource development quarterly, v. 26, n. 2, Summer, 2015.

MENDES FILHO, E. Uma avaliação do programa de apoio ao arranjo produtivo local da pedra Cariri (CE). 2009. 103f. Dissertação (Mestrado em Economia) Universidade Federal do Ceará, Fortaleza, Ceará, 2009.

MERCADO BRASILEIRO DE SOFTWARE: PANORAMA E TENDÊNCIAS- 1a․ ed. São Paulo: ABES - Associação Brasileira das Empresas de Software, 2016.

MESSENGER. Disponível em: <https://www.messenger.com/>. Acesso em: 21 out. 2018.

MOZZATO, A. R; BITENCOURT, C. C. Understanding Interorganizational Learning Based on Social Spaces and Learning Episodes. BAR, Rio de Janeiro, v. 11, n. 3, art. 3, pp. 284-301, July/Sept. 2014.

NEGREIROS, M. do M. D. Uso corporativo de mídias sociais digitais para a gestão de pessoas e gestão de conhecimento em restaurantes na cidade do Natal/RN. 2015, 98 f. Dissertação ( Mestrado em administração) - Programa de pósgraduação em administração - ppga, Universidade Potiguar, Natal, 2015.

NEUMAN, W. L. Social research methods, qualitative and quantitative approaches (3rd ed.). Boston: Allyn and Bacon, 1997.

OURO FILHO, A. M. do; OLAVE, M. E. L.; BARRETO, I. D. de C. Fatores Desarticuladores da Cooperação em Arranjos Produtivos Locais: Um Estudo Quantitativo no APL de Confecções de Tobias Barreto/SE. Brazilian Business Review-BBR, Vitória-ES,v.12, n.5, , Set.- Out. 2015.

PANJAITAN, M. J.; NOORDERHAVEN, Ni. G. Trust, Calculation, and Interorganizational Learning of Tacit Knowledge: An Organizational Roles Perspective. Organization Studies, v. 30 (10), p. 1021-1044.

PANJAITANA, M. J.; NOORDERHAVENB, N. G. Formal and informal interorganizational learning within strategic alliances. Research Policy, v. 37, p. 1337-1355, 2008.

PARK, S.; STYLIANOU, A.; SUBRAMANIAM, C.; NIU, Y. Information technology and interorganizational learning: An investigation of knowledge exploration and exploitation processes. Information \& Management, v. 52, p. 998-1011, 2015.

PINTO, H; CRUZ, A. R.; COMBE, C. Cooperation and the emergence of maritime clusters in the Atlantic: Analysis and implications of innovation and human capital for blue growth. Marine Policy, v. 57, p. 167-177, 2015.

REDE DE PESQUISA EM SISTEMAS E ARRANJOS PRODUTIVOS E INATIVOS LOCAIS (Brasil). Foco. 2013. Disponível em: <http://www.redesist.ie.ufrj.br/>. Acesso em: 05 dez. 2016. 
SÁNCHEZ, R. G.; MUIÑA, F. E. G. La creación de redes de cooperación entre empresarias rurales a través de las TIC: el caso de la plataforma ARTEMUR (España). Revista Sociedad y Economía, n. 21, p. 149-168, 2011.

SCOTT, J. Facilitating Interorganizational Learning with Informatio in Technology. Journal of Management Information Systems, v.17, n. 2, p. 81-113.

SIE, R. L.L; RIJPKEMA, M. B.; STOYANOV, S.; SLOEP, P. B. Factors that influence cooperation in networks for innovation and learning. Computers in Human Behavior, v. 37, p. 377-384, 2014.

SILVA, P. N.; MUYLDER, C. F. De. Inteligência competitiva e cooperação na percepção dos atores do arranjo produtivo local de software da Região Metropolitana de Belo Horizonte. Perspectivas em Ciência da Informação, v.20, n.2, p.134-157, abr./jun. 2015.

SIMON KEMP; WE ARE SOCIAL. Digital in 2016. Disponível em: < https://d1 ri6y1 vinkzt0.cloudfront.net/media/documents/We\%20Ares\%20Social\%20Di gital\%20in\%202016v02-160126235031.pdf>. Acesso em 14 dez. 2016.

THE WHATSAPP NATION. Mobile Ecosystem Fórum. Disponível em: < http://mobileecosystemforum.com/2016/07/13/the-WhatsApp-nation/>. Acesso em 22 dez. 2016.

TISOTT, P. B.; TOMIELO, T.; KROTH, D. F.; OLEA, P. M.; BORELLI, V. Alice; NESPOLO, D. O arranjo produtivo local - tecnologia da informação da serra gaúcha como um sistema de inovação. Inteligência Competitiva, São Paulo, v. 6, n. 6, p. 2547, jan./mar. 2016.

VASUDEVA, G.; ALEXANDER, E. A.; JONES, S. L. Institutional Logics and Interorganizational Learning in Technological Arenas: Evidence from Standard-Setting Organizations in the Mobile Handset Industry. OrganizationScience, v. 26, n. 3, pp. 830-846, May-June, 2015.

VERSCHOORE, J. R.; BALESTRIN, A. Ganhos competitivos das empresas em redes de cooperação. R. Administração - Eletrônica, São Paulo, v.1, n.1, art.2, jan./jun. 2008.

YANG, S. M.; FANG, S. C.; FANG, S. R.; CHOU, C. H. Knowledge exchange and knowledge protection in interorganizational learning: The ambidexterity perspective. Industrial Marketing Management, v. 43, p. 346-358, 2014.

YIN, R. K. Estudo de caso: planejamento e métodos. 5. Ed. Porto Alegre: Bookman, 2015.

ZAMBRANA, A. de A.; TEIXEIRA, R. M. Governança e suas implicações na promoção da cooperação em APLs: evidências em Aracaju/SE. Organizaçõ̃es em contexto, São Bernardo do Campo, v. 12, n. 23, jan.-jun. 2016. 